\title{
Seeing and Overcoming the Complexities of Intersectionality
}

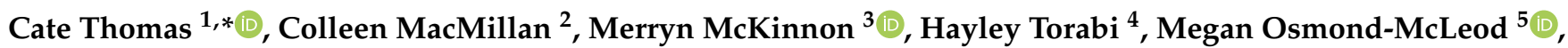 \\ Ellen Swavley ${ }^{6}$, Tamzen Armer ${ }^{7}$ and Kimberley Doyle ${ }^{1}$
}

1 Faculty of Science, Charles Sturt University, 2678 Wagga Wagga, Australia; kidoyle@csu.edu.au

2 Agriculture \& Food, CSIRO, 2601 Canberra, Australia; colleen.macmillan@csiro.au

3 National Centre for the Public Awareness of Science, Australian National University, 2601 Canberra, Australia; merryn.mckinnon@anu.edu.au

4 Human Resources Division, Australian National University, 2601 Canberra, Australia; hayley.torabi@anu.edu.au

5 Diversity Inclusion Belonging, CSIRO, 2113 North Ryde, Australia; megan.osmond@csiro.au

6 People \& Diversity, University of Canberra, 2617 Canberra, Australia; ellen.swavley@canberra.edu.au

7 Science in Australia Gender Equity (SAGE), 2601 Canberra, Australia;

tamzen.armer@sciencegenderequity.org.au

* Correspondence: cthomas@csu.edu.au; Tel.: +61-407-312-741

check for

updates

Citation: Thomas, C.; MacMillan, C.; McKinnon, M.; Torabi, H.; Osmond-McLeod, M.; Swavley, E.; Armer, T.; Doyle, K. Seeing and Overcoming the Complexities of Intersectionality. Challenges 2021, 12, 5. https://doi.org/10.3390/ challe12010005

Received: 8 January 2021

Accepted: 1 February 2021

Published: 5 February 2021

Publisher's Note: MDPI stays neutral with regard to jurisdictional claims in published maps and institutional affiliations.

Copyright: (c) 2021 by the authors. Licensee MDPI, Basel, Switzerland. This article is an open access article distributed under the terms and conditions of the Creative Commons Attribution (CC BY) license (https:// creativecommons.org/licenses/by/ $4.0 /)$.

\begin{abstract}
Background: Intersectionality contests that individuals have multiple characteristics in their identity that cannot be siloed or deemed exclusive to each other. Understanding and utilising an intersectional lens in organisations can increase inclusion of individuals and organisational performance. An educational package known as the Intersectionality Walk (IW) was developed by the authors, piloted, and evaluated in order to break down the commonly held descriptors of diversity silos that fragments inclusion, and to understand how various identity characteristics compound disadvantage. The paper outlines the need to transition from siloed views of diversity to a more intrinsic view of identity to achieve inclusivity. Methods: The IW was developed and trialled with a series of work-based scenarios and realistic multifaceted personas. Data collection occurred preand post-IW utilising a mixed methods approach. Responses to Likert scale surveys and open-ended questions were captured and analysed via inductive and grounded theory perspectives. Results: An improved awareness and understanding of individual knowledge, reflectivity and positionality relating to intersectionality and intersectional approaches was reported on completion of the IW. Furthermore, responses reported how and why organisations can approach and improve inclusivity via using intersectional approaches. Conclusions: The IW as an educational package has a positive impact and is a key linkage for all employers to build an inclusive culture and to harness the talent of all employees. Further research will occur to measure the implemented change in organisations following the IW.
\end{abstract}

Keywords: intersectionality; gender equity; Intersectionality Walk; organisational change; inclusion; strategy; STEMM; diversity

\section{Introduction}

Intersectionality as a concept is growing and literature has captured the discourse of intersectionality theory as a catalyst for social change and intersectionality as the roots of activism [1-3]. However, the evaluation of intersectionality strategies or those strategies using an intersectional lens to transform organisational behaviour and culture is extremely limited, with even fewer evaluations occurring on what works in the Science Technology Engineering Mathematics and Medicine (STEMM) field [4,5]. This paper focuses on the nexus of gender equity issues in STEMM organisations and intersectionality; that gender itself intersects with other forms of inequity, oppression and disadvantage [6-8]. The importance of evaluating the development and introduction of intersectionality education, with respect to positive impact on individuals as future change-makers within organisations 
is also discussed. This paper highlights through original research why intersectionality is important in redressing simplistic views to inform positive social change at the individual, structural and organisational levels [9].

Intersectionality as a concept was introduced by Kimberlé Crenshaw in 1989 to disrupt the ideologies of how inequalities manifest as distinct silos of individuals by gender or by race rather than considering how different inequalities intersect, compound, and are mutually constitutive rather than mutually exclusive $[10,11]$. Intersectionality was originally based in feminism and critical race studies and now is being more widely applied at the individual, structural and political levels, and across disciplines [11-16]. As Bowleg [10] (p. 1267) articulates, "Intersectionality is a theoretical framework for understanding how multiple social identities such as race, gender, sexual orientation, socio-economic status, and disability intersect at the micro level of individual experience to reflect interlocking systems of privilege and oppression (i.e., racism, sexism, heterosexism, classism) at the macro social structural level". No longer can we work to a notion of universality or traditional thinking around inclusion and diversity [17] but instead we must work from a framework of intersectionality built on understanding how the broader context of identity impacts on individuals differently, at different times and in different contexts. Through understanding intersectionality, we can examine how vulnerable populations, for example women of minority backgrounds, have additional facets to their identities or extra challenges to contend with including racism, racialised sexism, ableism, ageism, classism, and homophobia or transphobia. Intersectionality may have similarity to diversity; however, intersectionality challenges the status-quo by taking a holistic approach to human individuality, explores new approaches, enhances understanding and encourages inclusivity at the micro, meso and macro levels $[10,18,19]$.

Through understanding intersectionality, we can acknowledge that gender diversity policies may have different outcomes for gender minorities of diverse backgrounds, which can be dependent on their levels of privilege [3,7]. Organisations that want to attract and retain gender minorities of diverse backgrounds must pay attention to and address this [18]. They may, however, be hindered first by a lack of shared and consistent understanding, and then by traditional thinking around inclusion strategies that too often unintentionally set symbolic boundaries around diversity groups [20]. To fully appreciate the vastness of all human talent, thinking, and knowledge (see Figure 1 below), organisations need a rethink to embrace intersectionality at an organisational and research level-so that systemic, structural, and policy processes and delivery are responsive and inclusive to all.

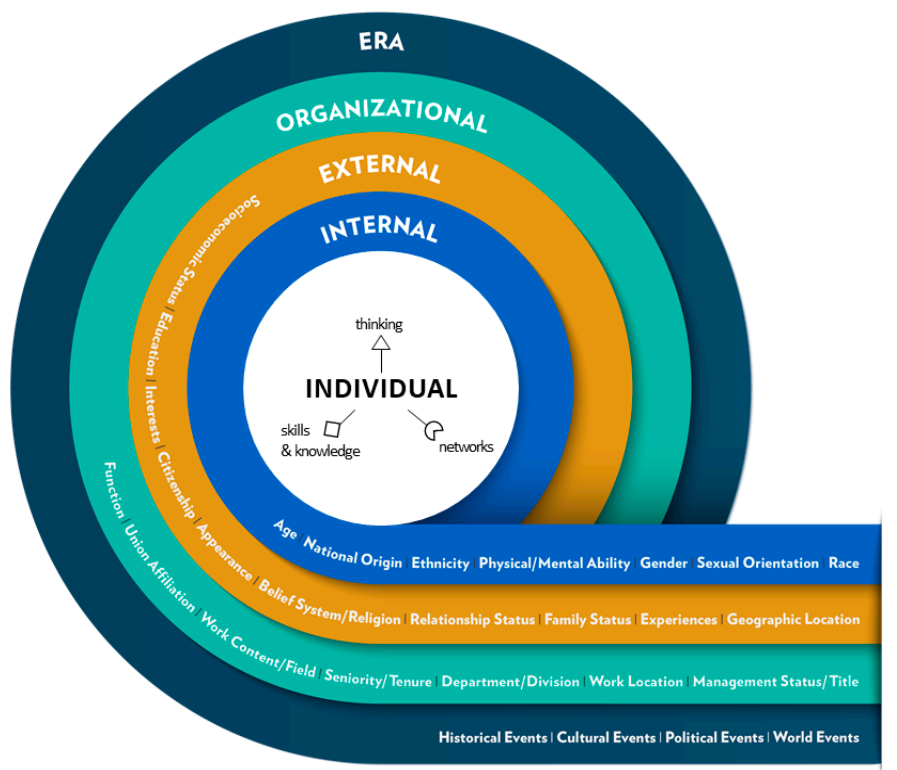

Figure 1. Individual's talent framework for intersectionality. 
As represented in Figure 1, an individual human holds intrinsic diversity of thinking, and acquired diversity of knowledge and skills, as well as networks, at any point in time. The intersections of an individual's gender, ethnicity, cultural background, linguistic background, age (and more) compound to create a unique individual with unique talents. This holds a wealth of potential for organisations, for innovation, and for valuing diverse individuals because it can expand enormously an organisation's quantum of diversity of thinking, knowledge and skills, and connectivity to diverse networks at multiple scales. There is a vast array of literature that discusses the various applications of an intersectional lens from a scholarly and research dimension. This literature provides insights into why intersectionality is important for understanding connections, client characteristics and behaviour, for service agencies for developing more holistic service provision, and for organisations in understanding and advancing equity [11,19,21-25]. It also recognises that intersectionality is not solely concerned with disadvantage. "What might seem like oppression in one setting can be experienced as privilege in another. Therefore, even oppression and privilege become contextual and relational" [18] (p.35). What is generally absent from the literature is the description of how to apply an intersectional lens through education and training to close the gap of a lack of knowledge on intersectionality, and why it is important for organisations including higher education institutions, to use intersectionality as a framework to provide environments where all individuals feel sought after for their talents and can thrive [26]. Applying intersectionality has the ability to disrupt and transform social inequalities and organisational rationale to go beyond traditional minority group thinking $[27,28]$. The application of an intersectional lens requires critical reflection and thinking in order to move away from traditional gendered organisational structures and processes, and to move beyond the status quo [6,7]. It challenges STEMM organisations and educational institutions to move beyond their traditional structures and processes, and to incorporate learning from other disciplines in order to be transformative. Organisational research and education that utilises an intersectional lens with practical actions is needed to challenge the views and values of organisations [23]. Research should question how practices, policy, and politics perpetuate inequity and inequality in order to re-focus on organisational social responsibility and to advance inclusion [23,29-31]. Organisations should not only respond to diversity and inequalities but rather embrace intersectionality to harness diverse talent, human capital and capability, and cultivate a sense of belonging for diverse individuals. This is imperative for facilitating change and understanding real inclusion in organisations and practice. In a case example, STEMM or science is not a single dimension and is moving away from a positivist approach to that of an intersectional approach, adopting an intersectional lens in research which provides a platform to reassess prior assumptions and identity formation, and to provide opportunities to redress inequalities such as sexism in science classrooms and labs [32,33].

The authors identified a need to go beyond discussing theoretical applications of intersectionality to developing an educational approach to take intersectionality from theory to practice and evaluate the impact of the application of intersectionality education [4]. It was recognised that an intersectional approach would facilitate a greater understanding of how multiple forms of discrimination may interact and compound to narrow STEMM contribution and participation at all stages of the 'STEMM education and career pathway'. Moving from intersectional theory to practice will assist in the development of a diverse and productive STEMM workforce operating at its full human potential. Key to this was the foundation of transformative learning and the building of an evidence base of practice promoting an intersectional view of inclusion, using an education module as a teaching moment pedagogy that required participants' critical self-reflection and self-evaluation of behaviours beyond the nominal universal diversity of identities [29]. The consideration of the importance and role of leadership is paramount for an intersectional focus to deliver change, not only by individual behavioural modelling, but by understanding disadvantage, and to challenge thinking, behaviour, structures, policy, systems and power $[20,30,34]$. The educational package was developed to place individuals, using personas, into the shoes of 
others who have different and potentially vulnerable identity characteristics. This was to challenge the participants' own concepts of intersectionality and to expand their thinking and logic of diversity and inclusion-to go beyond their own world view [8]. The shared recognition of the impact intersectionality has on employees in the workplace and testing of real-time mitigation strategies described here can then be used to enhance the capability of organisations to achieve gender equity outcomes.

The educational package developed was a set of resources, to equip staff members within institutions and organisations to facilitate an "Intersectionality Walk" (IW) - an authentic and experiential activity for a participating group of 10-15 people, to broaden their understanding and appreciation of the impact of intersectionality in the workplace. The IW as an exercise provides a practical demonstration of the compounding effect of multiple factors of identity that can disadvantage people in the workplace. It is an action-based activity on how intersectionality affects an individual's engagement at work, and how identification and mitigation of structural barriers can change this. It also deliberately includes an educational component on how to achieve impactful structural change-through inclusion.

The set of resources developed - the "Intersectionality Walk Pack" —include a briefing video providing an overview of intersectionality in the context of Australia's Women in the STEM Decadal Plan and the UK's Athena SWAN Principles; a guide to facilitating the IW; and a downloadable pack with personas and scenarios, ready to pick up and use within any organisation (see Supplementary Materials). There is also a "Virtual Intersectionality Walk Tracker" available, to facilitate the IW in online settings.

The IW is conducted in a circle of between 6 and 15 people. Multiple circles can be accommodated provided there is sufficient room and facilitators. Each of the circles are led by a facilitator who hands out personas and reads out scenarios which will determine the steps taken by participants. The personas with identities/characteristics and 'realistic workplace scenarios' cover a range of gender identities and vulnerability characteristics. Participants take on a persona, then take one or more steps backwards in each scenario, depending on the compounding factors that impact on their persona in that scenario. If the IW is being conducted virtually, the participants keep a tally of how many virtual steps they have taken. At the completion of the IW, feedback from participants is sought about the changes that could be made to the workplace scenarios to be more inclusive for their persona. The facilitator incorporates the suggested changes into the scenarios and re-runs the IW to assess the effect of the changes.

Example scenario: there is a task requiring after hours work, for at least a couple of weeks, over the next 6 months-the exact timing is not known. The team working on the task, are all "Aussie", white and male. Your manager has asked for more volunteers and has offered a trip to a high-profile conference as an incentive. You have always wanted to have the opportunity to attend this event.

Do you volunteer for the after-hours work? Do you feel your contributions are valued?

Example persona: $\mathrm{Li}$ is a female research academic. She is from a non-English-speaking background and does not have any family in the country. She is married with a full-time employed spouse, and she is in the late stages of pregnancy.

In the above example, we can see how Li could be impacted by multiple factors of her identity. The team is predominately white, male, and Australian-born. Li, as a woman from a non-English speaking background, may feel less inclined to volunteer for the task. Further, being pregnant and with no family in the country, she is unlikely to be able to attend the high-profile conference, or volunteer for after-hours work. In this instance, we can see how Li would take multiple steps away from the inner circle, and how this demonstrates to participants the compounding effect of intersectionality in the workplace.

The IW was used to research the understanding of the theory and practice of intersectionality and inclusion at the individual, career, and institutional levels. Both qualitative and quantitative data were obtained and analysed, with a focus on the individual and institutional scales. Here we report the findings of this research and discuss the implications of the IW as an educational resource for enabling meaningful and structural inclusive change 
in diversity and inclusion practices that take a holistic approach to individuals and for realising the benefits for organisations.

\section{Materials and Methods}

\subsection{Evaluation Data Collection}

Evaluation data were collected via a pre- and post-questionnaire with the IW participants. Ethical approval for the study was provided by the Australian National University (2020/005) and Charles Sturt University (H20357) Human Research Ethics Committees (National Statement on Ethical Conduct in Human Research 2007). The IW was first trialled at the "Catalysing Gender Equity 2020 Conference" held in February 2020 in Adelaide, Australia. Participants registered to attend the session three weeks prior to the conference and upon registration were sent an invitation to participate in the study, an information sheet and a link to the online questionnaire. Participation in the study was not a requirement for participating in the IW Workshop at the conference.

The questionnaire, hosted on Qualtrics ${ }^{\mathrm{XM}}$, comprised closed and open-ended questions that aimed to identify how well participants felt they understood intersectionality and its impacts on careers, and how well equipped they perceived themselves and their workplaces to respond to intersectional issues. Questions specifically asked how they would define intersectionality, what they felt were important factors in intersectionality (for example religion, gender, sexual orientation, marital status), whether they agreed they had a personal role to play in promoting inclusive practices and policies, and which characteristics of intersectionality were important at their institution. They were asked to reflect upon whether their workplaces recognised or addressed intersectionality and what structural barriers may exist. Participants were also asked to reflect upon whether they felt intersectionality issues had influenced their career, positively or negatively. Responses to the questionnaires were submitted before the IW session at the conference, with each participant using a unique identifier consisting of their mother's maiden name and their date of birth. These identifiers were used to match the pre- and post-IW responses. Demographic information was specifically not collected in the questionnaires, as will be discussed in Section 2.2.

The week following the conference, all registered attendees were sent an email with a link to the follow-up questionnaire. It asked many of the same questions regarding personal understanding of intersectionality and its impacts as described above, how to respond appropriately to intersectional issues and to again reflect on whether their organisation recognised and/or addressed intersectionality and associated structural barriers. It also asked if any element of the IW changed their perception of intersectionality, positively or negatively, and to comment on the most and least useful parts of the IW for them.

\subsection{Data Analysis}

The data have been analysed purely in terms of IW participants, rather than along traditional demographic lines, in acknowledgement of the fundamental concept of intersectionality as demonstrated by Figure 1. An individual is more than the sum of the identifiable 'demographic' categories. The IW aims to demonstrate that two people sharing a common demographic or even two or three characteristics do not translate into experiencing the workplace (and indeed the world) in a similar way. Similarly, the authors would not want to infer that any singular demographic cohort is in fact homogenous and, therefore, conclude that responses are indicative of that characteristic.

The open-ended responses were collated and coded by one author. After preliminary coding by a single author, the code categories were examined and confirmed by the other study authors, with all authors in agreement of the categories used in analysis. For the question about whether participants felt their careers had been influenced positively or negatively by intersectional issues, responses were initially coded into pre-determined categories of yes, no, and unsure. The main reasons for participants' answers to this question were not categorized and are presented verbatim. In the analysis of the responses 
describing the structural barriers to addressing intersectionality within institutions, the code categories were derived from a close reading of all the words in responses [35] which were then grouped into similar themes through an inductive process of category development [36]. Given the limited amount of research that exists about understanding and the lived experience of intersectionality, an inductive approach to category development was deemed most appropriate [37]. The key words and phrases specific to each category are provided in Table 1.

Table 1. Categories and exemplary phrases and words used in qualitative data analysis.

\begin{tabular}{|c|c|c|}
\hline Question & Code Categories & Exemplary Text \\
\hline \multirow{7}{*}{$\begin{array}{l}\text { Thinking about your workplace, what } \\
\text { structural barriers need redressing to } \\
\text { allow any implemented practice, policy, } \\
\text { organisational systems and/or cultural } \\
\text { change to effectively mitigate any } \\
\text { disadvantage created by intersectionality? }\end{array}$} & Lack of accountability & $\begin{array}{c}\text { There is no accountability because Human } \\
\text { Resources (HR) is not impartial ... }\end{array}$ \\
\hline & Lack of awareness & $\begin{array}{l}\text { First, understanding of the impact ... } \\
\ldots \text { ignorance could be redressed ... }\end{array}$ \\
\hline & Lack of data & $\begin{array}{c}\text { We need national data at granular scales on } \\
\text { what barriers intersectional people } \\
\text { experience... }\end{array}$ \\
\hline & Lack of leadership & $\begin{array}{c}\text { Leading by example to promote cultural } \\
\text { change ... }\end{array}$ \\
\hline & Lack of policy & Policies \\
\hline & Lack of resources & $\begin{array}{l}\text { The biggest problem at my institution is the } \\
\text { scarcity of resources ... }\end{array}$ \\
\hline & Multiple & $\begin{array}{c}\text { Goodness me, see our Science in Gender } \\
\text { Equity Australia (SAGE) Action Plan as a } \\
\text { starting point! }\end{array}$ \\
\hline \multirow{4}{*}{$\begin{array}{c}\text { What kind of evidence would indicate to } \\
\text { you that these barriers have been } \\
\text { addressed? }\end{array}$} & Data & $\begin{array}{l}\text { Measurable success indicators, KPI's or the } \\
\text { like ... }\end{array}$ \\
\hline & Inclusive practice & $\begin{array}{l}\text { No missed meetings due to caring } \\
\text { responsibilities }\end{array}$ \\
\hline & Increased awareness & $\begin{array}{c}\text { Training and conversations at senior levels } \\
\text { about structures of privilege and } \\
\text { disadvantage ... }\end{array}$ \\
\hline & Increased diversity & $\begin{array}{l}\text { Retention and promotion of minority } \\
\text { groups... }\end{array}$ \\
\hline
\end{tabular}

This project used a grounded theory approach, which allows for patterns to emerge from the data. Grounded theory has evolved from its initial conception by Glasser and Strauss in 1967 with other key theorist such as Corbin, Clarke and Charmaz approaching data collection and analysis differently [38]. There have been many uses of grounded theory in applied research including studies in the organisational culture and development sphere since the 1970s [39]. In this research, the authors utilised grounded theory as a constructivist form of inquiry "in which the researcher derives a general, abstract theory of a process, action, or interaction grounded in the views of participants. This process involves using multiple stages of data collection and the refinement and interrelationship of categories of information" [40] (p. 13). Given there is little known about the understanding and lived experience of intersectionality of individuals within Australian institutions, using an inductive approach [41] such as grounded theory enabled the use of the stories and experiences of participants to identify meaning, develop emerging themes and establish a preliminary theoretical framework [42]. In turn, these were used to identify potential paths for further analysis. As articulated by Ebrahimi, grounded theory when utilised as an applied research tool, provides the opportunity to bring educational and practice 
researchers together and has the benefit of assisting participants themselves to manage their own responses to current and future challenges that may arise within organisations [39].

Pseudonyms are used in the presentation of results.

\section{Results}

3.1. Quantitative Assessment of Understanding of Theory and Practice of Intersectionality and Inclusion at the Individual and Institutional Levels

Participant quantitative responses to the pre- and post-IW sliding-scale survey questions were analysed. The key results were that initially there was limited understanding of intersectionality and how to practice inclusion, and that the IW improved participants understanding of both conceptual and practical aspects of intersectionality. More than a third of respondents $(36 \%)$ reported not having a good understanding of intersectionality prior to the IW; however, after the IW $100 \%$ of respondents said they agreed/strongly agreed that they had a good understanding of intersectionality (Figure 2). In terms of individual practice, a third of respondents reported not knowing how to use inclusive behaviours and practices prior to the IW; however, after the IW $100 \%$ of respondents reported that they knew how to use inclusive behaviours and practices (Figure 2). Almost half of participants were not aware of institutional strategies to change structural barriers hindering intersectionality prior to the IW, while after the IW 88\% of respondents reported that they had awareness of strategies to utilise toward structural change at the institutional level (Figure 3).

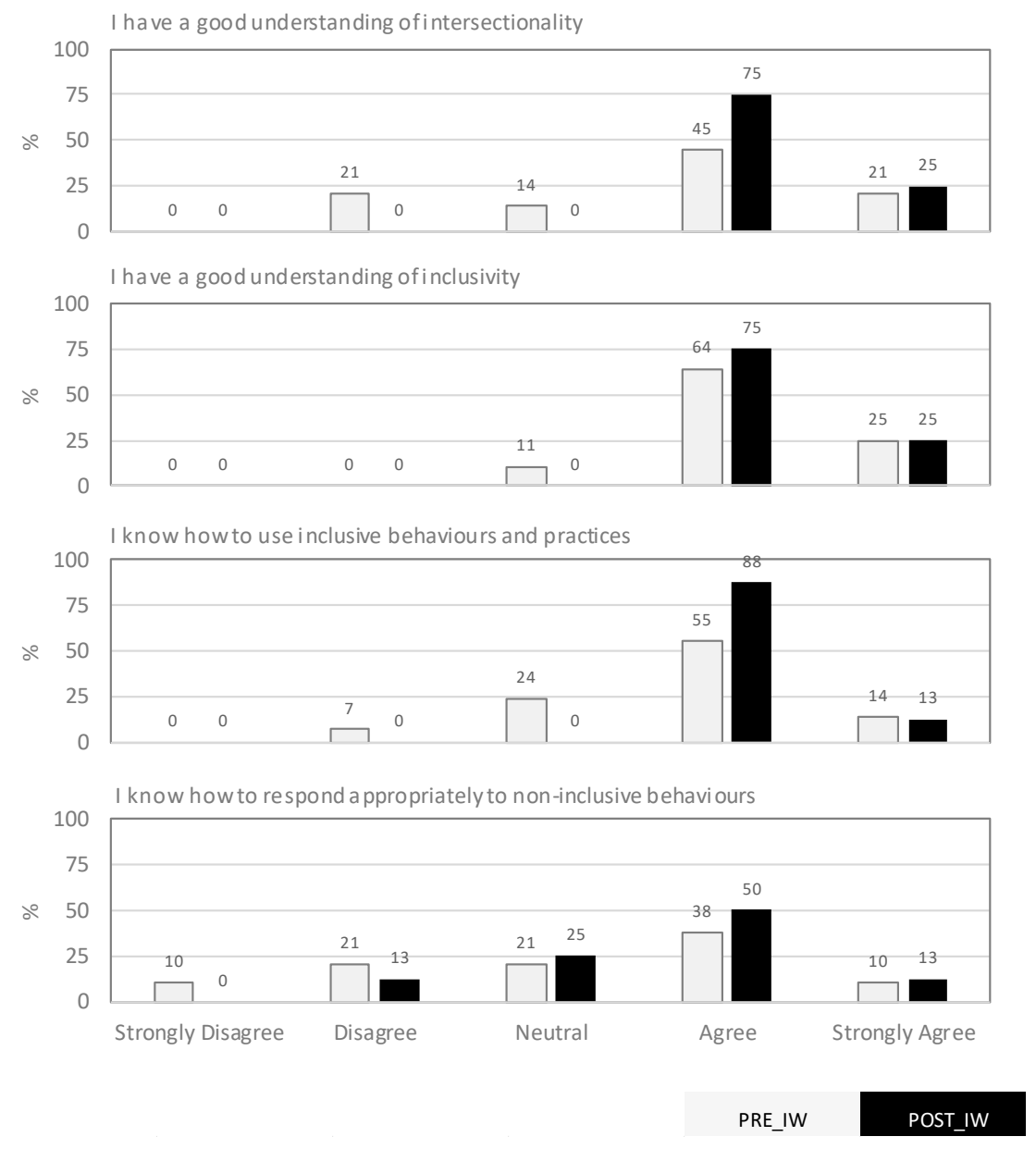

Figure 2. Intersectionality Walk participant survey responses as individuals, before and after the IW. Survey responses are shown as \% positive responses for each category. PRE_IW denotes before the IW $(\mathrm{N}=29)$, POST_IW denotes after the IW $(\mathrm{N}=8)$. 


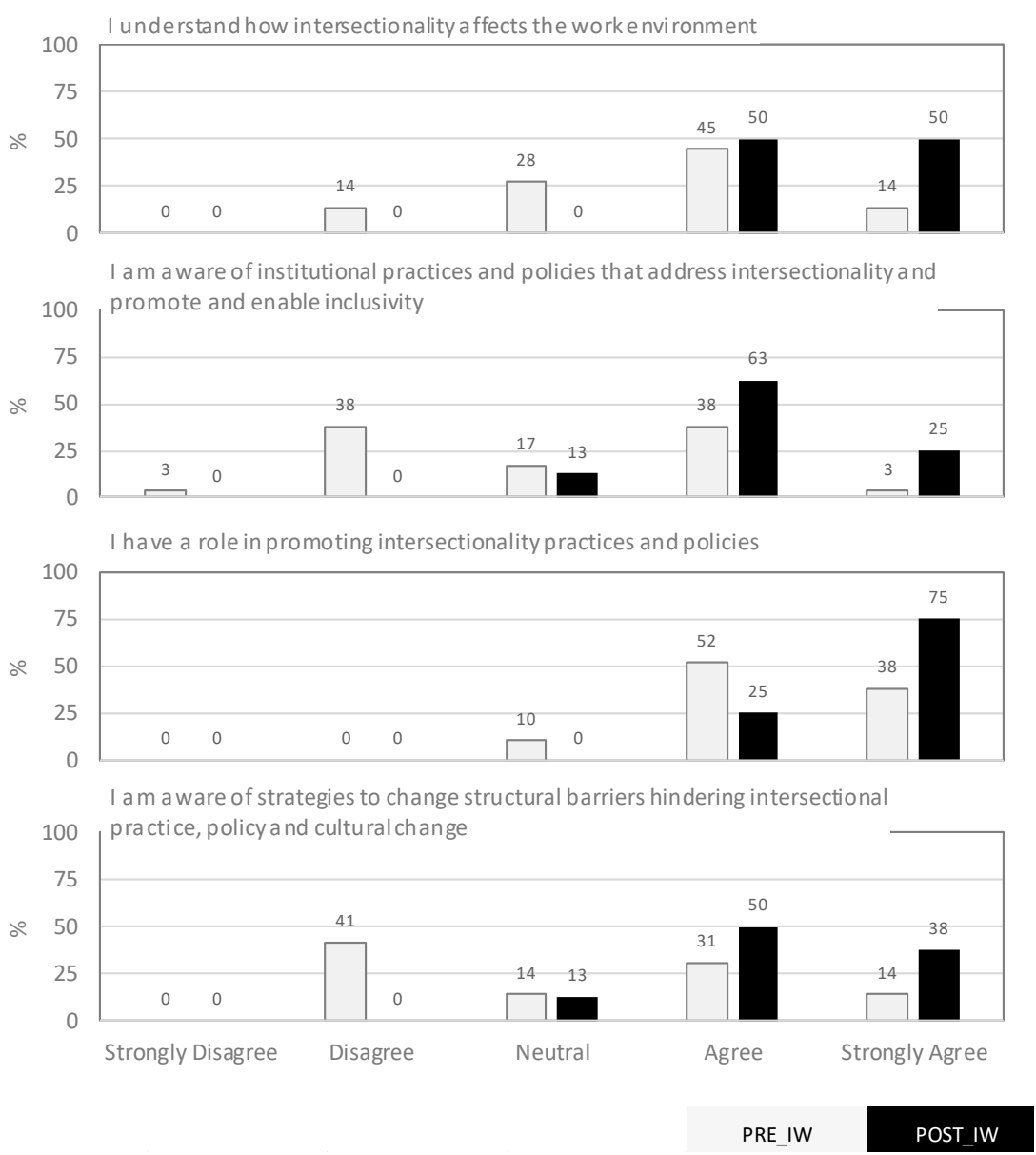

Figure 3. Intersectionality Walk participant survey responses reflection their view of institutions, before and after the IW. Survey responses are shown as \% positive responses for each category. PRE_IW denotes before the IW $(\mathrm{N}=29)$, POST_IW denotes after the IW $(\mathrm{N}=8)$.

\subsection{Qualtitative Results}

A total of nine matched (pre/post) complete responses were received and retained for qualitative analysis. All respondents were asked whether they felt intersectional issues had impacted their careers, positively or negatively. Four respondents indicated that they felt they had, with the impact considered negative for all but one respondent who felt there were positive and negative aspects:

“My direct experience of shame and stigma has given me great insight into parallel experiences of discrimination. I have done a lot of work advocating for reducing boundaries to participating and diffusing the access to power." (Sam, pre-IW response)

One participant did not feel that they were affected and three were unsure if they were impacted or not. It was among these three participants that the greatest changes were observed in the post-IW responses. Eden was not sure if intersectional issues had affected her in the pre-IW survey or if they had, it was largely positive: "Privilege of being an educated white woman". Post-workshop, her answer was still a maybe, but her interpretation of the impacts had changed to negative: "Caring responsibilities for my three children meant that I stepped away from the paid workforce for 10 years".

Similarly, Riley showed a stark difference in how she perceived intersectional issues had affected her when comparing her pre- and post-responses:

"As a young woman with no caring responsibilities, I have experienced gendered stereotypes and the related barriers, but most of my colleagues think I'm white so I don't think I've had too many race/cultural background issues." (Riley, pre-IW) 
"As a young white-presenting woman without caring responsibilities I am able to do things like go to conferences, travel for workshops, and network internationally, which opens up many opportunities that wouldn't otherwise exist. (Riley, post-IW)

Riley's pre-workshop response seems to imply that because she could 'hide' an aspect of her identity, she felt she was not adversely affected. Post-workshop, the notion of 'hiding' aspects of her identity is still apparent; however, she believes not having some of the other intersectional identities such as mother or care-giver actually provides her with some benefit.

Participants were asked before and after the IW about the structural barriers to addressing intersectional issues in their workplaces. Almost all of the participants identified barriers which arose due to a 'lack of ...' something. In the pre-IW survey, a lack of awareness was the most commonly identified barrier $(n=4)$. This lack of awareness was paired with a lack of policies by one respondent, and a lack of resources by another whose comment exemplifies the nature of most answers to this question:

"The biggest problem at my institution is the scarcity of resources and the belief that equity work is not worth the cost - this is exacerbated for thinking about how various inequities intersect." (Reid, pre-IW)

In the post-IW survey, four of the respondents retained elements of their pre-IW answers, namely regarding a lack of awareness $(n=1)$, policy $(n=1)$ and resources $(n=2)$. Five respondents changed their answers entirely from awareness and accountability to a lack of data $(n=2)$, a lack of leadership $(n=2)$ and one respondent changing from a lack of accountability to identifying multiple barriers:

"Too many to warrant discussion here-not much is in place to address any issues although there are some things in place to improve support of women" (Peta, post-IW)

In both pre- and post-IW surveys, the need for data was a recurring theme both as a structural barrier and as evidence that barriers had been addressed. Of the four respondents who listed data, each was consistent in highlighting the need for long-term data collection both to help overcome structural barriers, and to indicate evidence that barriers had been successfully addressed as exemplified in Jo's responses:

"I think the key structural barriers are about monitoring and evaluating our diversity, and discrimination. In order to know what's wrong in the system, we first need to measure it-and have the accurate and comprehensive, and transparent measures in place." (Jo, post-IW, structural barrier)

"An evidence-based monitoring and evaluation measure (MERI) that uses accurate language and looks at all aspects of human diversity and discrimination." (Jo, post-IW survey, evidence that barriers had been addressed)

\section{Discussion}

The results from the study signified that participants, by undertaking the educational IW, had an improved understanding of, firstly, what intersectionality is and, secondly, how to bring about structural change at the individual and institutional levels.

In addition to improving understanding, participating in the IW also influenced some participants' perception of how issues of intersectionality may have influenced their careers-positively or negatively — consistent with the idea of oppression and privilege being contextual [18]. What was surprising was that this pilot study found evidence of people choosing which identities to 'make public' in a professional setting, through the responses of one participant (Riley). Perhaps it is not so surprising that someone would choose to obscure their cultural background (or gender identity or disability or any other identity) to avoid additional barriers to professional progress, but rather that evidence of it was found so readily. This highlights systemic issues that illustrate, even within those with a demonstrated interest in Gender Equity Diversity Inclusion (GEDI), organisations are not 
yet creating environments where people feel their talents are the most important factor in their career.

One key finding from this study is the importance of data, with the lack of awareness, resources and tools to collect data related to intersectionality consistently cited as barriers to progress and the presence of intersectionality data considered an indicator of success. As this study has shown, even those with a vested interest in intersectional issues are approaching the problem with an imperfect understanding of what intersectionality is, let alone how they may facilitate meaningful inclusion at an individual or organisational level. In order to create truly inclusive workplaces, we need data. Data will enable us to develop an accurate description of where we are starting from, set realistic and achievable targets, and to identify potential impediments on the path of progress. In considering the approach to the educational IW, the deliberate use of personas and carefully crafted scenarios was instrumental to evoke positive change. The use of personas provided the opportunity for participants to 'think' and 'be' outside their normal practice and behaviour, and thus the personas were successful in expanding the individual's worldview. The personas and scenarios were tools for reflexive practice for each individual-to experience something they previously had not experienced, that is, the 'as close to' real-world lived experiences of others. This practice also aids in a richness and depth of empathy towards others. The use of personas is an evidence-based pedagogy for adult learners to experience difference and to form empathy for others $[43,44]$. What this demonstrates is how 'experiencing' catalyses reflection and change. By asking participants to 'step into the shoes of others' they gained deeper personal reflection and insights as cited in the qualitative results section. This level of reflection and change would likely not occur for participants in a lecture style professional development.

In the case of the IW, the personas and scenarios did impact on the self-reporting improvement of awareness and understanding. Even though the responses pre- and postIW did show self-reporting improvement had occurred, at this stage the authors have not progressed data collection to the point of knowing whether that self-reflection of increased awareness actually translated into changed behaviour and implementation of strategy. Further research design and data collection will occur to allow this.

The data and results are positive; however, it must also be noted that the impact of the level of awareness in the cohort potentially underestimates the impact of the IW (preand post-knowledge/awareness). Why? By virtue of their attendance at a gender equity conference, the individuals in this cohort all had a general understanding of gender equity and related issues and were predominately from this field of study; this could suggest an underestimation of the potential impact of this educational tool on those in other fields. As the IW continues to roll out across the Australian Higher Education and Research sector and other key partners, data will be analysed by cohort, and across cohorts, to learn how the IW can be best targeted to reach its full potential.

More education to translate theory to practice is needed. As we witness the term intersectionality being used more frequently, it is now critical that shifts occur for deeper understanding of what it actually is, how it translates and applies to real-world structures, 'exclusion' and 'inclusion', and how to practically change the system through expansive inclusion to realise value at the individual and institutional levels. Measuring what people understand intersectionality to be-such as in the pre-IW used here-is important and useful. It can identify the maturity levels of a group of individuals or even an institution. Only through reflective education such as the IW will change occur.

Responses and questions arose from the IW and data collection as to what extent are people hiding/minimising/obscuring elements of their identity in order to not be disadvantaged in their career and being excluded. The IW provides an opportunity for education to redress a lack of lived experience or awareness. Using personas and scenarios highlights, for example, the potential negative impacts of being a woman from a minority background on a career trajectory; often such impacts are not recognised until pointed out. Working in a siloed diversity framework will not lead to change. Applying an 
intersectional lens demonstrates the need for an intrinsic view of human identity. Until such time as policy and organisational mantras move away from the siloing effect of diversity characteristics, and move to applying an intersectional lens, the ability of individuals to fully contribute their talents to organisations will not be realised. What the IW has demonstrated is that it provides an opportunity for organisations to consider how various characteristics intersect to compound both/either disadvantage and/or privilege, and to respond. The IW (and applying an intersectional lens) provides a ground-breaking linkage to organisational performance and culture if applied as an educational tool to modify behaviour and thinking.

\subsection{Limitations and Future Work}

This was a pilot study. All of the participants were at a gender equity and inclusion conference and so likely had a higher-than-average understanding of intersectionality and engagement in diversity and inclusion work. Scaling to a larger cohort of organisational leaders and staff may allow a more robust assessment into the effectiveness of the IW in translating intersectional theory to practice. It may also give insight into potential knowledge gaps between leaders, who often have greater access to development supporting organisational diversity policies and aspirations, and general staff, who typically are offered less developmental support but are nevertheless asked to implement and support those policies and aspirations in their day-to-day work and interactions. Inviting organisational policy-makers, as opposed to leaders, to participate and comparing pre- and post-IW responses and proposed mitigation strategies may further reveal the impact of perceived structural barriers at the levels and roles where policies are shaped and implemented and where translation of aspiration to implementation can often be frustratingly incremental. The need to measure progress is clear and is the next step for the authors. This gap in the data is due to the infancy of the roll out of the educational IW; however, we cannot evaluate progress (or absence thereof) unless we measure.

\subsection{Further Research}

As previously indicated, further research and data collection is required to measure the impact of education on the development of organisational systems to respond, that is, the increased awareness and understanding of intersectionality within the organisational environment. The challenge is moving the data collection from a pre- and post- IW scenario to include a more longitudinal aspect to benchmark and measure organisational change.

\section{Conclusions}

The IW as an educational initiative has demonstrated its impact to increase awareness and understanding of intersectionality and inclusion. It is potentially a tool for all employers to build an inclusive and responsive culture, and to harness the talent of all employees; the counter scenario is that when institutions do not take a holistic approach to individuals who are diverse and experience intersectional disadvantage, then there is a loss of talent from institutions that limits the competitive edge of an organisation. An individual's increase in awareness, and understanding of intersectionality, empathy for others, and the acknowledgement of one's own role in promoting intersectional practice, lead to positive changes in redressing to institutional structural barriers, systems, and cultures. In order to achieve structural change, inclusion is key; however, this requires education and strategies with an intersectional lens to be employed in all aspects of the organisation. The IW educational package is a ground-breaking linkage for practice, performance and culture, and has been achieved through the careful and purposeful pedagogical approaches chosen. The preliminary pilot data indicate the IW educational package has merit and that data capture is imperative to measure changes to awareness and understanding of intersectional practices that promote structures, systems and cultures which ultimately advance inclusion. The research that was undertaken and reported is the catalyst for further 
work and longitudinal evaluation of the impact of the IW educational package on change within organisations.

Supplementary Materials: The Intersectionality Walk resources are available online at https: / www. sciencegenderequity.org.au/resources/.

Author Contributions: Conceptualization of paper, C.T.; Methodology, C.T.; M.M. and C.M.; Formal Analysis, C.M. and M.M.; Investigation, C.T.; C.M. and M.M.; Resources, C.M.; C.T.; M.M. and M.O.-M. Data Curation, M.M.; Writing-Original Draft Preparation, C.T.; M.M.; C.M. and H.T.; Writing-Review and Editing, C.T.; M.M.; C.M.; H.T.; K.D.; T.A.; M.O.-M. and E.S. All authors have read and agreed to the published version of the manuscript.

Funding: This research received no external funding.

Institutional Review Board Statement: The study was conducted according to the National Statement on Ethical Conduct in Human Research 2007 guidelines (National Health and Medical Research Council Act 1992) and approved by the Australian National University (2020/005) and Charles Sturt University (H20357) Human Research Ethics Committees.

Informed Consent Statement: Informed consent was obtained from all subjects involved in the study.

Data Availability Statement: Data may be made available upon reasonable request from the corresponding author.

Acknowledgments: The authors wish to acknowledge the support of Science in Australia Gender Equity for their support in progressing the issue of intersectionality, diversity and inclusivity. We also acknowledge the early contributions of Kerry Elliott (CSIRO) in helping to shape the direction of the IW.

Conflicts of Interest: The authors declare no conflict of interest.

\section{References}

1. Carastathis, A. The Concept of Intersectionality in Feminist Theory. Philos. Compass 2014, 9, 304-314. [CrossRef]

2. Knapp, G.-A. Intersectional Invisibility: Inquiries into a Concept of Intersectional Studies, in Framing Intersectionality: Debates on a Multi-Faceted Concept in Gender Studies; Lutz, H., Vivar, M.T.H., Supik, L., Eds.; Routledge: London, UK, 2011 ; pp. 187-205.

3. Severs, E.; Celis, K.; Erzeel, S. Power, privilege and disadvantage: Intersectionality theory and political representation. Politics 2016, 36, 346-354. [CrossRef]

4. Muntinga, M.E.; Krajenbrink, V.Q.E.; Peerdeman, S.M.; Croiset, G.; Verdonk, M.P. Toward diversity-responsive medical education: Taking an intersectionality-based approach to a curriculum evaluation. Adv. Health Sci. Educ. Theory Pract. 2015, 21, 541-559. [CrossRef]

5. McKinnon, M. The absence of evidence of the effectiveness of Australian gender equity in STEM initiatives. Aust. J. Soc. Issues 2020, 1-14. [CrossRef]

6. Acker, J. Gendered organizations and intersectionality: Problems and possibilities. Equal. Divers. Incl. Int. J. 2012, 31, 214-224. [CrossRef]

7. Dennissen, M.H.J.; Benschop, Y.W.M.; Brink, M.C.L.v.d. Rethinking diversity management: An intersectional analysis of diversity networks. Organ. Stud. 2020, 41, 219-240. [CrossRef]

8. Manyweathers, J.; Lymn, J.; Rurenga, G.; Murrell-Orgill, K.; Cameron, S.; Thomas, C. The Lived Experience of Gender and Gender Equity Policies at a Regional Australian University. Soc. Sci. 2020, 9, 115. [CrossRef]

9. Shields, S.A. Gender: An Intersectionality Perspective. Sex Roles 2008, 59, 301-311. [CrossRef]

10. Bowleg, L. The Problem with the Phrase Women and Minorities: Intersectionality-An Important Theoretical Framework for Public Health. Am. J. Public Health 2012, 102, 1267-1273. [CrossRef]

11. Cho, S.; Crenshaw, K.W.; McCall, L. Toward a Field of Intersectionality Studies: Theory, Applications, and Praxis. Signs J. Women Cult. Soc. 2013, 38, 785-810. [CrossRef]

12. Bilge, S. Intersectionality Undone: Intersectionality from Feminist Intersectionality Studies. Du Bois Rev. 2013, 10, 405-424. [CrossRef]

13. Corus, C.; Saatcioglu, B. An intersectionality framework for transformative services research. Serv. Ind. J. 2015, 35, 415-429. [CrossRef]

14. Dhamoon, R.K. Considerations on Mainstreaming Intersectionality. Political Res. Q. 2010, 64, 230-243. [CrossRef]

15. Green, M.A.; Evans, C.R.; Subramanian, S.V. Can intersectionality theory enrich population health research? Soc. Sci. Med. 2017, 178, 214-216. [CrossRef] [PubMed]

16. Shaw, L.R.; Chan, F.; McMahon, B.T. Intersectionality and Disability Harassment: The Interactive Effects of Disability, Race, Age, and Gender. Rehabil. Couns. Bull. 2012, 55, 82-91. [CrossRef] 
17. Thomas, G.; Macnab, N. Intersectionality, diversity, community and inclusion: Untangling the knots. Int. J. Incl. Educ. 2019, 1-18. [CrossRef]

18. Marfelt, M.M. Grounded intersectionality. Equal. Divers. Incl. Int. J. 2016, 35, 31-47. [CrossRef]

19. Ruel, S. The "silent killers" of a STEM-professional woman's career. Equal. Divers. Incl. Int. J. 2018, 37, 728-748. [CrossRef]

20. Vallas, S.; Cummins, E. Relational Models of Organizational Inequalities: Emerging Approaches and Conceptual Dilemmas. Am. Behav. Sci. 2014, 58, 228-255. [CrossRef]

21. Al-Faham, H.; Davis, A.M.; Ernst, R. Intersectionality: From Theory to Practice. Annu. Rev. Law Soc. Sci. 2019, 15, 247-265. [CrossRef]

22. Hankivsky, O.; Grace, D.; Hunting, G.; Giesbrecht, M.; Fridkin, A.; Rudrum, S.; Ferlatte, O.; Clark, N. An intersectionality-based policy analysis framework: Critical reflections on a methodology for advancing equity. Int. J. Equity Health 2014, 13, 119. [CrossRef]

23. Heiskanen, T.; Leinonen, M.; Ylöstalo, H.; Otonkorpi-Lehtoranta, K. Gender Issues on Change Agenda-Practising Intersectionality in Action Research. Gend. Work Organ. 2018, 25, 459-474. [CrossRef]

24. Liu, H. Re-radicalising intersectionality in organisation studies. Ephemera 2018, 18, 81-101.

25. Ruiz Ben, E. Intersectionality in the Practice of Mixed Methods Gender Research. J. Res. Gend. Stud. 2018, 8, 73-88.

26. Carver, P. Mission Statements-Adopting Intersectionality as a Framework in Higher Education. J. Bus. Divers. 2020, $20,17-27$.

27. Núñez, A.-M.; Rivera, J.; Hallmark, T. Applying an intersectionality lens to expand equity in the geosciences. J. Geosci. Educ. 2020, 68, 97-114. [CrossRef]

28. Steinfield, L.; Sanghvi, M.; Zayer, L.T.; Coleman, C.A.; Ourahmoune, N.; Harrison, R.L.; Hein, W.; Brace-Govan, J. Transformative intersectionality: Moving business towards a critical praxis. J. Bus. Res. 2019, 100, 366-375. [CrossRef]

29. Azzopardi, C. Cross-Cultural Social Work: A Critical Approach to Teaching and Learning to Work Effectively across Intersectional Identities. Br. J. Soc. Work 2020, 50, 464-482. [CrossRef]

30. Pullen, A.; Rhodes, C.; McEwen, C.; Liu, H. Radical politics, intersectionality and leadership for diversity in organizations. Manag. Decis. 2019, 1-14. [CrossRef]

31. Thomas, J.; Thomas, C.; Smith, K. The challenges for gender equity and women in leadership in a distributed university in regional Australia. Soc. Sci. 2019, 8, 165. [CrossRef]

32. Avraamidou, L. Science identity as a landscape of becoming: Rethinking recognition and emotions through an intersectionality lens. Cult. Stud. Sci. Educ. 2020, 15, 323-345. [CrossRef]

33. Viruell-Fuentes, E.A.; Miranda, P.Y.; Abdulrahim, S. More than culture: Structural racism, intersectionality theory, and immigrant health. Soc. Sci. Med. 2012, 75, 2099-2106. [CrossRef] [PubMed]

34. Hendricks, K.; Deal, N.; Mills, A.J.; Mills, J.H. Intersectionality as a matter of time. Manag. Decis. 2020, 1-16. [CrossRef]

35. Miles, M.B.; Huberman, A.M. Qualitative Data Analysis: An Expanded Sourcebook, 2nd ed.; Sage: Newbury Park, CA, USA, 1994.

36. Mayring, P. Qualitative Content Analysis [28 paragraphs]. Forum Qual. Soc. Res. 2000, 1, Art.20.

37. Elo, S.; Kyngäs, H. The qualitative content analysis process. J. Adv. Nurs. 2008, 62, 107-115. [CrossRef]

38. Ratnapalan, S. Qualitative approaches: Variations of grounded theory methodology. Can. Fam. Phys. 2019, 65, 667-668.

39. Ebrahimi, M. The Grounded Theory Methodology in Organization Studies within Qualitative Research, in Qualitative Exploration of Grounded Theory in Organizational Research; IGI Global: Hershey, PA, USA, 2020; pp. 1-27.

40. Creswell, J.W.; Creswell, J.D. Research Design: Qualitative, Quantitative, and Mixed Method Approaches, 5th ed.; SAGE: Los Angeles, CA, USA, 2018.

41. Alston, M.; Bowles, W. Research for Social Workers: An Introduction to Methods, 4th ed.; Bowles, W., Ed.; Allen \& Unwin: Sydney, Australia, 2018.

42. Creswell, J.W.; Hanson, W.E.; Plano, V.L.C.; Morales, A. Qualitative Research Designs. Couns. Psychol. 2016, 35, $236-264$. [CrossRef]

43. Canham, S.L.; Mahmood, A. The use of personas in gerontological education. Gerontol. Geriatr. Educ. 2018, 40, 468-479. [CrossRef]

44. Zagallo, P.; McCourt, J.; Idsardi, R.; Smith, M.K.; Urban-Lurain, M.; Andrews, T.C.; Haudek, K.; Knight, J.K.; Merrill, J.; Nehm, R.; et al. Through the Eyes of Faculty: Using Personas as a Tool for Learner-Centered Professional Development. CBE Life Sci. Educ. 2019, 18, ar62. [CrossRef] 